\title{
Clinical Study \\ Differences in the Form of Presentation between Papillary Microcarcinomas and Papillary Carcinomas of Larger Size
}

\author{
Carles Zafon, ${ }^{1}$ Juan Antonio Baena, ${ }^{2}$ Josep Castellví, ${ }^{3}$ Gabriel Obiols, ${ }^{1}$ Gabriela Monroy, ${ }^{1}$ \\ and Jordi Mesa ${ }^{1}$
}

${ }^{1}$ Department of Endocrinology, Hospital General Universitari Vall d'Hebron, Pg. Vall d'Hebron 119-129, 08035 Barcelona, Spain
${ }^{2}$ Department of Surgery, Unit of endocrinological surgery, Hospital General Universitari Vall d'Hebron, 08035 Barcelona, Spain
${ }^{3}$ Department of Pathology, Hospital General Universitari Vall d'Hebron, 08035 Barcelona, Spain

Correspondence should be addressed to Carles Zafon,26276czl@comb.cat

Received 12 August 2010; Revised 20 October 2010; Accepted 18 November 2010

Academic Editor: Maria João M. Bugalho

Copyright (c) 2011 Carles Zafon et al. This is an open access article distributed under the Creative Commons Attribution License, which permits unrestricted use, distribution, and reproduction in any medium, provided the original work is properly cited.

\begin{abstract}
Papillary thyroid carcinomas (PTCs) with a diameter $\leq 1 \mathrm{~cm}$ are referred to as papillary microcarcinomas (PTMCs). The prognostic factors for PTMCs have not been defined. Different clinical and histopathologic variables were studied in 152 PTCs, including 74 PTMCs and 78 PTCs of larger size. We found that PTMCs are associated with less multifocality $(P=.046)$ and bilaterality $(P=.003)$, fewer lymphadenectomies $(P<.001)$, and a higher rate of incidental tumours $(P<.001)$. Moreover, patients with a low aggressive profile were significantly older than the remaining patients $(54 \pm 13.7$ years versus $45.8 \pm 13.1$ years; $P=.001)$. In conclusion PTMCs show significant differences compared to PTCs of larger size in the form of presentation. Furthermore, it is possible that the classic risk factors, which are well validated in PTCs, such as age, must be cautiously interpreted in the current increasing subgroup of PTMCs.
\end{abstract}

\section{Introduction}

It has been clearly demonstrated that there is an increasing worldwide incidence of papillary thyroid carcinomas (PTCs). It is uncertain whether this is a real phenomenon, or whether it is due to an increased rate of detection [1]. Practices for management of thyroid diseases have changed over the past few decades. The wide availability of ultrasound (US) and fine needle aspiration biopsy (FNAB) and the improved accuracy of histopathologic examination of surgical specimens have been suggested to be reasons for the increased rate of detection. Moreover, among the new cases, the highest incidence has been observed in the smallest tumors [2]. In the USA, $49 \%$ of the increased incidence of PTCs consisted of cancers measuring $\leq 1 \mathrm{~cm} \mathrm{[3].} \mathrm{In} \mathrm{Europe,} \mathrm{Leenhardt} \mathrm{et} \mathrm{al.} \mathrm{[4]}$ reported that the proportion of tumors of this size increased from $18.4 \%$ between 1983 and 1987 to $43.1 \%$ between 1998 and 2001 [4]. Similar results have been confirmed by other authors worldwide $[2,5-8]$.

PTCs measuring $\leq 1 \mathrm{~cm}$ are referred to as papillary thyroid microcarcinomas (PTMCs) [9]. Although PTMCs are not recognized as a specific entity in the tumour, node, and metastasis (TNM) classification, PTMCs are considered a subset of PTCs that exhibit a more benign behavior. PTMCs usually follow an indolent course and carry an excellent prognosis. Distant metastases and mortality rates are reported to be $<0.5 \%$ for PTMCs [10]. Two large series have recently confirmed the excellent prognosis for PTMCs in long-term followup $[5,11]$. Nevertheless, some authors suggest that there exist a subgroup of PTMCs that can be aggressive, requiring therapeutic management similar to larger tumors [12]. Thus, no agreement has been reached about the optimal treatment of PTMCs. In recent years, several clinical and histologic risk factors for aggressiveness have been identified in PTMCs, such as size $\leq 5 \mathrm{~mm}$, multifocality, capsular invasion, tumor extension beyond the parenchyma, lymph node involvement, and the extent of primary surgery [12-17]. In contrast, some studies have failed to identify independent prognostic factors, arguing that to distinguish PTCs on the basis of size alone may be clinically irrelevant $[18,19]$. Because PTMCs are being diagnosed with increasing frequency, identification of specific prognostic factors is of outmost importance. 
In the present study, we describe the clinical and pathologic presentation of PTMCs, compared with papillary thyroid carcinomas of larger size (LPTCs). We have analyzed the classic risk factors and studied the clinical and histologic characteristics present at the time of diagnosis which were associated with a higher risk of recurrence in PTMCs, such as multifocality, lymph node metastases, and mode of detection (incidental versus nonincidental tumors).

\section{Methods}

PTMCs were defined as PTCs measuring $\leq 1 \mathrm{~cm}$ in greatest diameter. Mode of detection refers to incidental (IPTMCs) or nonincidental tumors (NIPTMCs). IPTMCs were identified in patients undergoing surgery for reasons unrelated to a thyroid malignancy, whereas patients with NIPTMCs underwent thyroidectomy for suspected malignancies. Multifocal disease was defined when $>1$ focus of PTCs was found in the thyroidectomy specimen. The following clinical variables were considered in the analysis: patient age, mode of detection, and extent of disease. The histopathologic variables after postoperative pathologic examination included the maximum diameter of the primary tumour, multifocality, bilaterality, extrathyroid extension, and lymph node metastases. Patients with PTMCs discovered incidentally, without multifocality, and without lymph node involvement were considered at low risk for developing recurrences. The confidentiality of patient information was absolutely maintained. Data are presented as the mean \pm SD. Statistical analysis was performed by Fisher's exact test for univariate analysis and by Student's t-test to compare continuous variables between groups. All tests were two-tailed. The levels of statistical significance are presented as $\mathrm{p}$ values. It was assumed that the observed differences were statistically significant at a $P<.05$ level.

\section{Results}

Between 2000 and 2009, 152 patients with PTCs were treated in our institution. Among these cases, there were 74 (48.7\%) PTMCs and 78 (51.3\%) LPTCs.

3.1. Microcarcinoma. The PTMC series included 59 females and 15 males (the female-to-male ratio was approximately 3.9). The mean age at the time of diagnosis was $50.1 \pm$ 13.2 years. Of the 74 cases, $67(90.5 \%)$ underwent total or near-total thyroidectomy, and only $7(9.5 \%)$ underwent lobectomies. The mean tumour size was $5.7 \pm 2.6 \mathrm{~mm}$. The pathology reports showed classic variant PTMCs in 64 patients $(86 \%)$, and follicular variants in 10 patients (14\%). Multifocal disease was documented in 26 patients (35.1\%). The patients with multifocal disease were younger than patients with a unique focus $(45.9 \pm 10.2$ years versus $52.5 \pm 14.2$ years; $P=.039$ ). Contralateral involvement was observed in 7 of 26 patients (27\%) with multifocal tumors. Regional lymph nodes were removed in 24 patients $(32.4 \%)$; of these, $12(50 \%)$ had nodal tumor involvement.
Overall, $72.2 \%$ of tumors ( 52 of 72 ) presented as IPTMCs (no information was available in 2 cases). In the other 20 cases $(27.8 \%)$, PTMCs were diagnosed by preoperative USguided FNABs. In patients with IPTMCs, the indications for surgery were as follows: 29 nontoxic multinodular goiters, 7 toxic multinodular goiters, and 16 solitary nodules. The difference in mean tumour size was statistically significant among the IPTMCs $(5 \pm 2.3 \mathrm{~mm})$ and NIPTMCs $(7.6 \pm$ $2.6 \mathrm{~mm} ; P<.001)$. Multifocality was present in $13(65 \%)$ of 20 patients classified as NIPTMCs, whereas multifocality was present in only $11(21 \%)$ of 52 patients with IPTMCs $(P<.001)$.

3.2. Larger Tumours. Tumors $>1 \mathrm{~cm}$ occurred in 62 females and 16 males (the female-to-male ratio was approximately 3.9). The mean age at the time of diagnosis was $46.2 \pm 14.1$ years. The primary surgical treatment consisted of total or near-total thyroidectomies in 76 patients and lobectomies in 2 patients. The mean tumor size was $25.21 \pm 11.8 \mathrm{~mm}$. The pathology reports showed classic variant LPTCS in 54 patients $(69.2 \%)$, follicular variant LPTCs in 21 patients (26.9\%), and one case each of columnar cell, cribiformmorular variant, and clear cell LPTCs. In this group, multifocality was found in 39 (50\%) samples. The age at presentation was not different in patients with and without multifocality. Contralateral involvement occurred in 25 of 39 patients $(64 \%)$ with multifocal tumours. Lymph node dissection was performed in 60 patients (77\%); of these, 36 patients $(60 \%)$ had nodal tumor involvement.

Only $11(15.5 \%)$ of 71 cases were classified as incidental tumors (no information was available in 7 cases). Of the indications for surgery were as follows: 7 nontoxic multinodular goiters, 3 solitary nodules, and 1 toxic multinodular goiter. Neither tumor size nor multifocality was significantly different among the incidental and nonincidental LPTCs.

3.3. PTMCs versus LPTCs. Table 1 shows the characteristics of both groups. Age and gender were not statistically different between the two groups of patients. However, based on the mode of presentation, patients with IPTMCs were significantly older than patients with incidental LPTCs $(51.9 \pm 13.5$ years versus $41.4 \pm 7.95$ years; $P=.016)$. Moreover, apart from size $(P<.0001)$, patients with PTMCs presented with multifocality $(P=.046)$ and bilaterality $(P=$ $.003)$ less often, fewer lymphadenectomies $(P<.001)$, and a higher rate of incidental tumours $(P<.001)$. In contrast, in patients in whom the lymph nodes were removed, there were no differences in the frequency of nodal metastases.

3.4. Aggressive Cases. Among all the 152 patients with PTCs, 40 patients $(26.3 \%)$ in whom PTMCs were discovered incidentally, without multifocality, and without lymph node involvement, were considered at a low risk for developing recurrences. These patients with a low aggressive profile were significantly older than the rest of the patients $(54 \pm 13.7$ years versus $45.8 \pm 13.1$ years; $P=.001)$. Moreover, the low aggressive profile was observed in $28(36.8 \%)$ of 76 patients $>45$ years of age and in $12(17.9 \%)$ of 67 patients $<45$ years 
TABLE 1: Differences between cases with papillary thyroid microcarcinomas (PTMCs) from those with papillary thyroid carcinomas of larger size (LPTCs).

\begin{tabular}{lccc}
\hline & PTMC $(n=74)$ & LPTC $(n=78)$ & $P$ \\
\hline Gender (female/male) & $15 / 59$ & $16 / 62$ & $\mathrm{~ns}$ \\
& $(79.7 \% / 20.3 \%)$ & $(79.5 \% / 20.5 \%)$ & \\
Age (y) & $50.17 \pm 13.22$ & $46.29 \pm 14.12$ & $\mathrm{~ns}$ \\
Size (mm) & $5.7 \pm 2.6$ & $25.2 \pm 11.8$ & $<.001$ \\
Multifocality & $26 / 74(35.1 \%)$ & $39 / 78(50 \%)$ & .046 \\
Lymphadenectomies & $24 / 74(32.4 \%)$ & $60 / 78(77 \%)$ & $<.001$ \\
Lymph M1 & $12 / 24(50 \%)$ & $36 / 60(60 \%)$ & $\mathrm{ns}$ \\
Incidental & $52 / 72(72.2 \%)$ & $11 / 72(15.2 \%)$ & $<.001$ \\
\hline
\end{tabular}

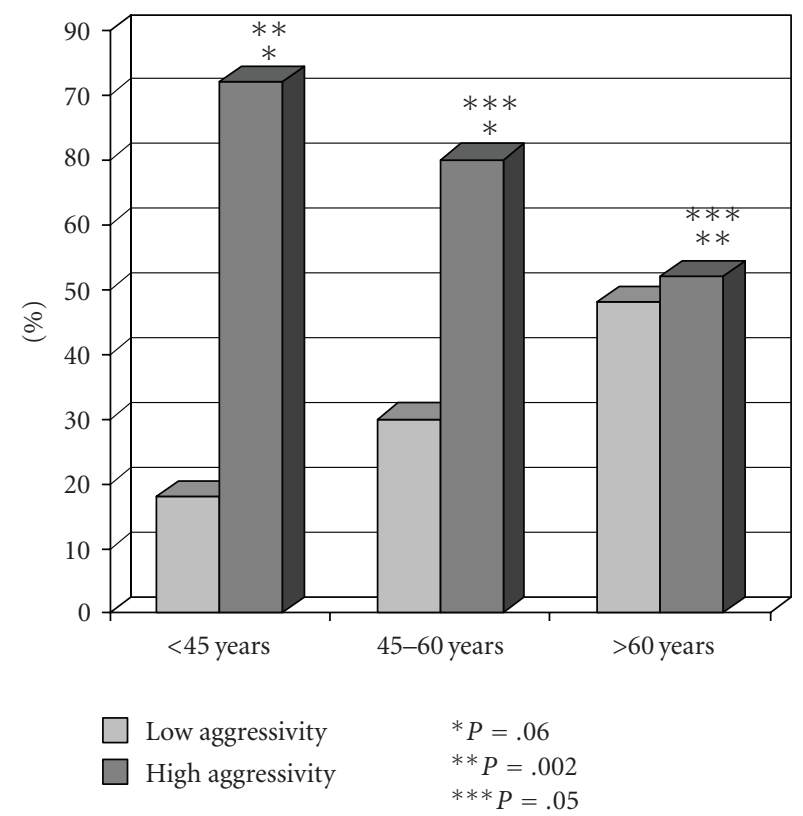

Figure 1: Patients with a low aggressive profile are significantly older than patients with a high aggressive profile.

of age $(P=.006)$. Finally, patients $\geq 60$ years of age had more cases with low aggressiveness compared to patients $<45$ years of age $(P=.002)$, and to patients between 45 and 60 years of age $(P=.05)$. This data is shown in Figure 1 .

\section{Discussion}

PTMC is defined as PTC measuring $\leq 1 \mathrm{~cm}$ in size [9]. This variant is also known as occult papillary carcinoma, latent papillary carcinoma, small papillary carcinoma, and papillary microtumor [20]. The current increase in incidence of PTC worldwide is mainly attributed to the corresponding increase in the diagnosis of PTMCs. In most recent series, especially the series that have analyzed cases from the last decade, PTMCs comprise nearly one-half of all the cases of PTCs [2, 3, 8, 21, 22]. Our series confirms this data. PTMCs are considered a subset of PTCs that exhibit a more benign behavior. Distant metastases and mortality rates are reported to be $<0.5 \%$ in patients with PTMCs [10].
Hay et al. [5] reported no difference between the observed number of deaths and the expected number of deaths in a cohort of 900 cases. Appetecchia et al. [23] reported that the outcome of PTMCs was favorable, even in the presence of lymph node metastases and local invasion. In contrast, some authors have suggested that there exist a subset of PTMCs that can be aggressive, requiring therapeutic management similar to larger PTCs $[6,12]$. Thus, no agreement has been reached about the optimal treatment of PTMCs. Some authors recommend an aggressive approach to PTMCs, while other authors suggest that no further treatment is needed after lobectomy or thyroidectomy. Moreover, it has even been proposed that observation without surgical treatment is appropriate [24].

Because the number of deaths is very small in patients with PTMCs, in the majority of series authors use the rate of recurrence as a marker of poor clinical outcome. Local and regional lymph node recurrences have been observed with a prevalence rate between $2 \%$ and 5.7\% [5, 25-27].

In recent years, some specific markers for aggressiveness have been identified [12-17]. Three of the most accepted factors are multifocality, lymph node metastasis, and the mode of diagnosis.

PTMCs frequently present as a multifocal process. Multiple foci are reported in approximately $7 \%-56 \%$ of cases $[5,6$, $10,28]$. A number of clinical studies have shown that patients with $\geq$ two foci had a higher recurrence rate and cancer mortality than those with unifocal PTMCs [5, 29]. Baudin et al. [30] reported that only two parameters influenced PTMC recurrences, one of which was multifocality. Moreover, multifocality has been associated with a high incidence of contralateral lobe involvement [31] and is an independent risk factor for metastases [32]. Hence, multifocal PTMCs have been considered to have a poor prognosis. In our series, we have detected a significantly higher rate of multifocality in LPTCs than in PTMCs.

PTMCs also show a high incidence of regional lymph node metastasis, occurring in $12 \%-64 \%$ of patients $[6,25$, 33-36]. Wada et al. [37] reported that $64.1 \%$ and $44.5 \%$ of patients have central and ipsilateral node involvement, respectively, and two-thirds of patients have lymph node metastasis in at least one of the two compartments. It has been described that cases with positive lymph nodes have a higher risk of recurrence [38]. Kim et al. [26] found that lateral cervical node metastasis was the most powerful independent predictor of clinical recurrence. However, other authors have reported that the outcome of PTMCs is favorable, even in the presence of lymph node metastases $[5,23,37,39]$. Prophylactic neck dissection is not routine in our hospital; node resection was not performed in the incidentally discovered cases. Therefore, the true number of positive lymph nodes is unknown; however, it is interesting to note that among patients in whom lymphadenectomy was performed, the rate of metastasis was not different between PTMCs and LPTCs.

Three circumstances may lead to the detection of a PTMC, as follows, PTMC found at autopsy, PTMC found incidentally in specimens of the thyroid removed for benign thyroid disease, and clinical PTMCs diagnosed before 
surgery [40]. Although the prevalence is highly variable, $>70 \%$ of PTMCs correspond to IPTMCs [10]. It has been suggested that clinical and biological behaviours may differ between IPTMCs and NIPTMCs [41, 42]. Some authors have found that overt tumors are associated with a higher incidence of multicentricity, extrathyroidal involvement, lymphovascular invasion, higher stage, risk of relapse, and death $[11,42-45]$. Hence, IPTMCs are associated with a better prognosis, whereas NIPTMCs may have more aggressive behavior. In like manner, we have found significant differences between both modes of presentation in relation to tumor size, multifocality, and age in the group of patients with PTMCs, whereas there were no such differences in tumors $>1 \mathrm{~cm}$ in size.

Age is considered to be the most important prognostic factor in PTCs and is included in all of the prognostic scoring systems. However, some investigators have failed to show that age affects the outcome of patients with PTMCs $[15,32,34$, $38,43,46,47]$. It is interesting to note that in our series, younger age is associated with a higher frequency of specific markers for aggressiveness. Thus, older patients have more IPTMCs without adverse markers, such as multifocality or lymph node metastases. Moreover, the group of patients $>60$ years of age has a higher incidence of cases with a lower risk of developing later recurrences than the rest of the patients.

In recent years, some of the molecules involved in neoplastic transformation have been explored as markers to assess the biological aggressiveness of PTMC [22, 48]. However, their use is at present not relevant to clinical decision making.

In summary, PTMCs exhibit significant differences in presentation from LPTCs. It is possible that the classic risk factors, which are well validated for PTCs, such as age, must be cautiously interpreted in the current increasing subgroup of PTMCs.

\section{Conflict of Interests}

The authors declare that they have no conflict of interests.

\section{References}

[1] S. Grodski and L. Delbridge, "An update on papillary microcarcinoma," Current Opinion in Oncology, vol. 21, no. 1, pp. $1-4,2009$.

[2] M. I. C. V. Cordioli, M. H. B. S. Canalli, and M. H. C. Coral, "Increase incidence of thyroid cancer in Florianopolis, Brazil: comparative study of diagnosed cases in 2000 and 2005," Arquivos Brasileiros de Endocrinologia e Metabologia, vol. 53, no. 4, pp. 453-460, 2009.

[3] L. Davies and H. G. Welch, "Increasing incidence of thyroid cancer in the United States, 1973-2002," Journal of the American Medical Association, vol. 295, no. 18, pp. 2164-2167, 2006.

[4] L. Leenhardt, P. Grosclaude, and L. Chérié-Challine, "Increased incidence of thyroid carcinoma in france: a true epidemic or thyroid nodule management effects? Report from the french thyroid cancer committee," Thyroid, vol. 14, no. 12, pp. 1056-1060, 2004.
[5] I. D. Hay, M. E. Hutchinson, T. Gonzalez-Losada et al., "Papillary thyroid microcarcinoma: a study of 900 cases observed in a 60-year period," Surgery, vol. 144, no. 6, pp. 980$988,2008$.

[6] J. Lee, Y. Rhee, S. Lee et al., "Frequent, aggressive behaviors of thyroid microcarcinomas in Korean patients," Endocrine Journal, vol. 53, no. 5, pp. 627-632, 2006.

[7] H. W. Lin and N. Bhattacharyya, "Survival impact of treatment options for papillary microcarcinoma of the thyroid," Laryngoscope, vol. 119, no. 10, pp. 1983-1987, 2009.

[8] A. Zengi, M. Karadeniz, M. Erdogan et al., "Does chernobyl accident have any effect on thyroid cancers in Turkey? A retrospective review of thyroid cancers from 1982 to 2006," Endocrine Journal, vol. 55, no. 2, pp. 325-330, 2008.

[9] R. Lloyd, R. De Lellis, P. Heitz et al., World Health Organization Classification of Tumors: Pathology and Genetics of Tumors of the Endocrine Organs, IARC Press, Lyon, France, 2004.

[10] E. Roti, E. C. degli Uberti, M. Bondanelli, and L. E. Braverman, "Thyroid papillary microcarcinoma: a descriptive and metaanalysis study," European Journal of Endocrinology, vol. 159, no. 6, pp. 659-673, 2008.

[11] S. Noguchi, H. Yamashita, S. Uchino, and S. Watanabe, "Papillary microcarcinoma," World Journal of Surgery, vol. 32, no. 5, pp. 747-753, 2008.

[12] C. Page, A. Biet, P. Boute, P. Cuvelier, and V. Strunski, "Aggressive papillary' thyroid microcarcinoma," European Archives of Oto-Rhino-Laryngology, vol. 266, no. 12, pp. 19591963, 2009.

[13] N. Ö. Küçük, P. Tari, E. Tokmak, and G. Aras, "Treatment for microcarcinoma of the thyroid-clinical experience," Clinical Nuclear Medicine, vol. 32, no. 4, pp. 279-281, 2007.

[14] S. H. Lee, S. S. Lee, S. M. Jin, J. H. Kim, and Y. S. Rho, "Predictive factors for central compartment lymph node metastasis in thyroid papillary microcarcinoma," Laryngoscope, vol. 118, no. 4, pp. 659-662, 2008.

[15] G. Mercante, A. Frasoldati, C. Pedroni et al., "Prognostic factors affecting neck lymph node recurrence and distant metastasis in papillary microcarcinoma of the thyroid: results of a study in 445 patients," Thyroid, vol. 19, no. 7, pp. 707-716, 2009.

[16] M. R. Pelizzo, I. M. Boschin, A. Toniato et al., "Papillary thyroid microcarcinoma (PTMC): prognostic factors, management and outcome in 403 patients," European Journal of Surgical Oncology, vol. 32, no. 10, pp. 1144-1148, 2006.

[17] E. Roti, R. Rossi, G. Trasforini et al., "Clinical and histological characteristics of papillary thyroid microcarcinoma: results of a retrospective study in 243 patients," Journal of Clinical Endocrinology and Metabolism, vol. 91, no. 6, pp. 2171-2178, 2006.

[18] N. Arora, H. K. Turbendian, M. A. Kato, T. A. Moo, R. Zarnegar, and T. J. Fahey, "Papillary thyroid carcinoma and microcarcinoma: is there a need to distinguish the two?" Thyroid, vol. 19, no. 5, pp. 473-477, 2009.

[19] C. Cappelli, M. Castellano, M. Braga et al., "Aggressiveness and outcome of papillary thyroid carcinoma (PTC) versus microcarcinoma (PMC): a mono-institutional experience," Journal of Surgical Oncology, vol. 95, no. 7, pp. 555-560, 2007.

[20] J. Rosai, V. A. LiVolsi, M. Sobrinho-Simoes, and E. D. Williams, "Renaming papillary microcarcinoma of the thyroid gland: the Porto proposal," International Journal of Surgical Pathology, vol. 11, no. 4, pp. 249-251, 2003.

[21] M. Colonna, A. V. Guizard, C. Schvartz et al., "A time trend analysis of papillary and follicular cancers as a function of tumour size: a study of data from six cancer registries in France 
(1983-2000)," European Journal of Cancer, vol. 43, no. 5, pp. 891-900, 2007.

[22] D. J. Lim, K. H. Baek, Y. S. Lee et al., "Clinical, histopathological, and molecular characteristics of papillary thyroid microcarcinoma," Thyroid, vol. 17, no. 9, pp. 883-888, 2007.

[23] M. Appetecchia, G. Scarcello, E. Pucci, and A. Procaccini, "Outcome after treatment of papillary thyroid microcarcinoma," Journal of Experimental and Clinical Cancer Research, vol. 21, no. 2, pp. 159-164, 2002.

[24] Y. Ito, T. Uruno, K. Nakano et al., "An observation trial without surgical treatment in patients with papillary microcarcinoma of the thyroid," Thyroid, vol. 13, no. 4, pp. 381-387, 2003.

[25] N. Besic, G. Pilko, R. Petric, M. Hocevar, and J. Zgajnar, "Papillary thyroid microcarcinoma: prognostic factors and treatment," Journal of Surgical Oncology, vol. 97, no. 3, pp. 221-225, 2008.

[26] T. Y. Kim, S. J. Hong, J. M. Kim et al., "Prognostic parameters for recurrence of papillary thyroid microcarcinoma," $B M C$ Cancer, vol. 8, article 296, 2008.

[27] M. R. Pelizzo, I. M. Boschin, A. Toniato et al., "Natural history, diagnosis, treatment and outcome of papillary thyroid microcarcinoma (PTMC): a mono-institutional 12-year experience," Nuclear Medicine Communications, vol. 25, no. 6, pp. 547-552, 2004.

[28] M. Dietlein, W. A. Luyken, H. Schicha, and A. LarenaAvellaneda, "Incidental multifocal papillary microcarcinomas of the thyroid: is subtotal thyroidectomy combined with radioiodine ablation enough?" Nuclear Medicine Communications, vol. 26, no. 1, pp. 3-8, 2005.

[29] J. D. Lin, T. C. Chao, C. Hsueh, and S. F. Kuo, "High recurrent rate of multicentric papillary thyroid carcinoma," Annals of Surgical Oncology, vol. 16, no. 9, pp. 2609-2616, 2009.

[30] E. Baudin, J. P. Travagli, J. Ropers et al., "Microcarcinoma of the thyroid gland the Gustave-Roussy Institute experience," Cancer, vol. 83, no. 3, pp. 553-559, 1998.

[31] E. Kim, T. Kim, J. Koh et al., "Completion thyroidectomy in patients with thyroid cancer who initially underwent unilateral operation," Clinical Endocrinology, vol. 61, no. 1, pp. 145-148, 2004.

[32] K. Gülben, U. Berberoğlu, O. Çelen, and H. H. Mersin, "Incidental papillary microcarcinoma of the thyroid-factors affecting lymph node metastasis," Langenbeck's Archives of Surgery, vol. 393, no. 1, pp. 25-29, 2008.

[33] S. Choi, T. Kim, J. Lee et al., "Is routine central neck dissection necessary for the treatment of papillary thyroid microcarcinoma?" Clinical and Experimental Otorhinolaryngology, vol. 1, pp. 41-45, 2008.

[34] Y. S. Chung, J. Y. Kim, JA. S. Bae et al., "Lateral lymph node metastasis in papillary thyroid carcinoma: results of therapeutic lymph node dissection," Thyroid, vol. 19, no. 3, pp. 241-246, 2009.

[35] Y. C. Lim, E. C. Choi, Y. H. Yoon, E. H. Kim, and B. S. Koo, "Central lymph node metastases in unilateral papillary thyroid microcarcinoma," British Journal of Surgery, vol. 96, no. 3, pp. 253-257, 2009.

[36] J. L. Roh, J. M. Kim, and C. I. Park, "Central cervical nodal metastasis from papillary thyroid microcarcinoma: pattern and factors predictive of nodal metastasis," Annals of Surgical Oncology, vol. 15, no. 9, pp. 2482-2486, 2008.

[37] N. Wada, Q. Y. Duh, K. Sugino et al., "Lymph node metastasis from 259 papillary thyroid microcarcinomas," Annals of Surgery, vol. 237, no. 3, pp. 399-407, 2003.

[38] S. M. Chow, S. C. K. Law, J. K. C. Chan, S. K. Au, S. Yau, and W. H. Lau, "Papillary microcarcinoma of the thyroid-prognostic significance of lymph node metastasis and multifocality," Cancer, vol. 98, no. 1, pp. 31-40, 2003.

[39] Y. Ito, C. Tomoda, T. Uruno et al., "Clinical significance of metastasis to the central compartment from papillary microcarcinoma of the thyroid," World Journal of Surgery, vol. 30, no. 1, pp. 91-99, 2006.

[40] K. Pazaitou-Panayiotou, M. Capezzone, and F. Pacini, "Clinical features and therapeutic implication of papillary thyroid microcarcinoma," Thyroid, vol. 17, no. 11, pp. 1085-1092, 2007.

[41] D. Barbaro, U. Simit, G. Meucci, P. Lapi, P. Orsini, and C. Pasquini, "Thyroid papillary cancers: microcarcinoma and carcinoma, incidental cancers and non-incidental cancersare they different diseases?" Clinical Endocrinology, vol. 63, no. 5, pp. 577-581, 2005.

[42] J. D. Lin, S. F. Kuo, T. C. Chao, and C. Hsueh, "Incidental and nonincidental papillary thyroid microcarcinoma," Annals of Surgical Oncology, vol. 15, no. 8, pp. 2287-2292, 2008.

[43] N. Besic, J. Zgajnar, M. Hocevar, and R. Petric, "Extent of thyroidectomy and lymphadenectomy in 254 patients with papillary thyroid microcarcinoma: a single-institution experience," Annals of Surgical Oncology, vol. 16, no. 4, pp. 920-928, 2009.

[44] C. Y. Lo, W. F. Chan, B. H. H. Lang, K. Y. Lam, and K. Y. Wan, "Papillary microcarcinoma: is there any difference between clinically overt and occult tumors?" World Journal of Surgery, vol. 30, no. 5, pp. 759-766, 2006.

[45] A. Pisanu, I. Reccia, O. Nardello, and A. Uccheddu, "Risk factors for nodal metastasis and recurrence among patients with papillary thyroid microcarcinoma: differences in clinical relevance between nonincidental and incidental tumors," World Journal of Surgery, vol. 33, no. 3, pp. 460-468, 2009.

[46] M. N. Pakdaman, L. Rochon, O. Gologan et al., "Incidence and histopathological behavior of papillary microcarcinomas: study of 429 cases," Otolaryngology-Head and Neck Surgery, vol. 139 , no. 5, pp. 718-722, 2008.

[47] G. Tzvetov, D. Hirsch, I. Shraga-Slutzky et al., "Welldifferentiated thyroid carcinoma: comparison of microscopic and macroscopic disease," Thyroid, vol. 19, no. 5, pp. 487-494, 2009.

[48] D. Cvejic, S. Selemetjev, S. Savin, I. Paunovic, I. Petrovic, and S. Tatic, "Apoptosis and proliferation related molecules (Bcl-2, Bax, p53, PCNA) in papillary microcarcinoma versus papillary carcinoma of the thyroid," Pathology, vol. 40, no. 5, pp. 475480, 2008. 


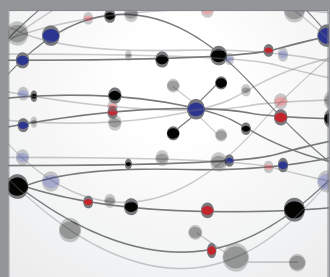

The Scientific World Journal
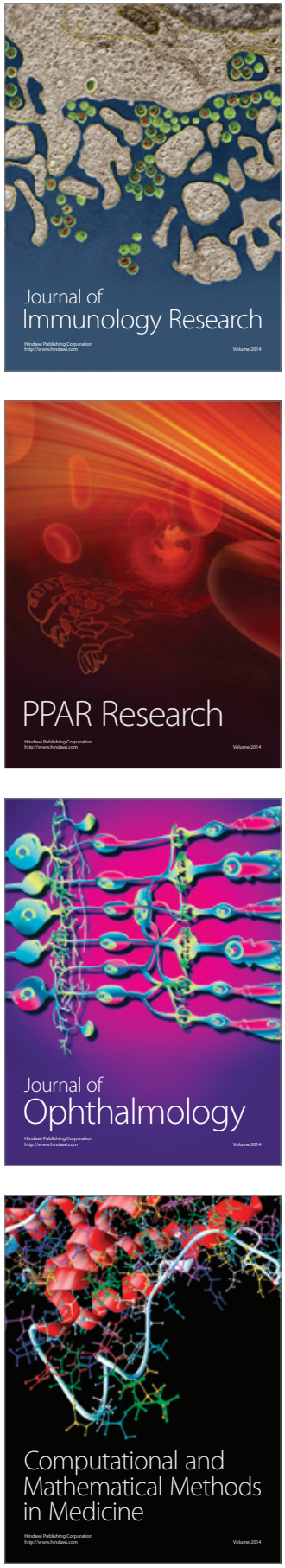

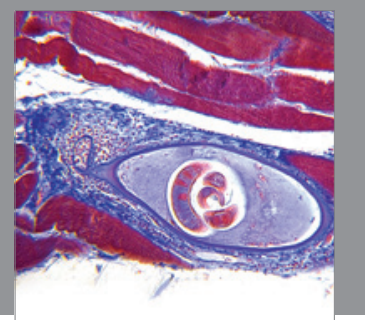

Gastroenterology

Research and Practice
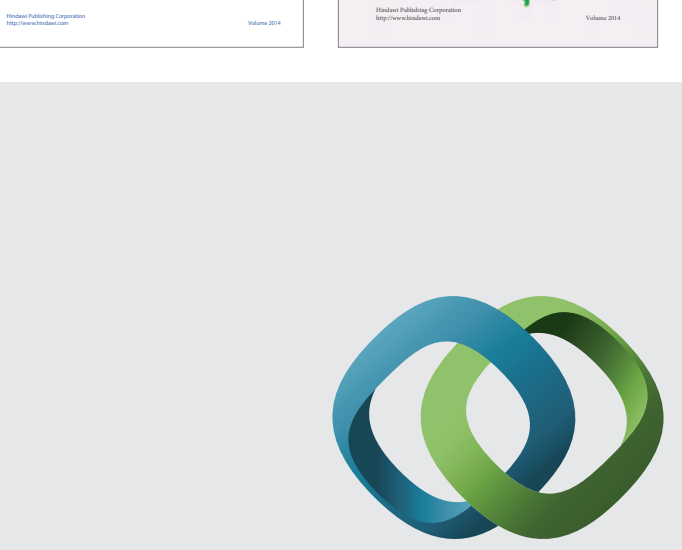

\section{Hindawi}

Submit your manuscripts at

http://www.hindawi.com
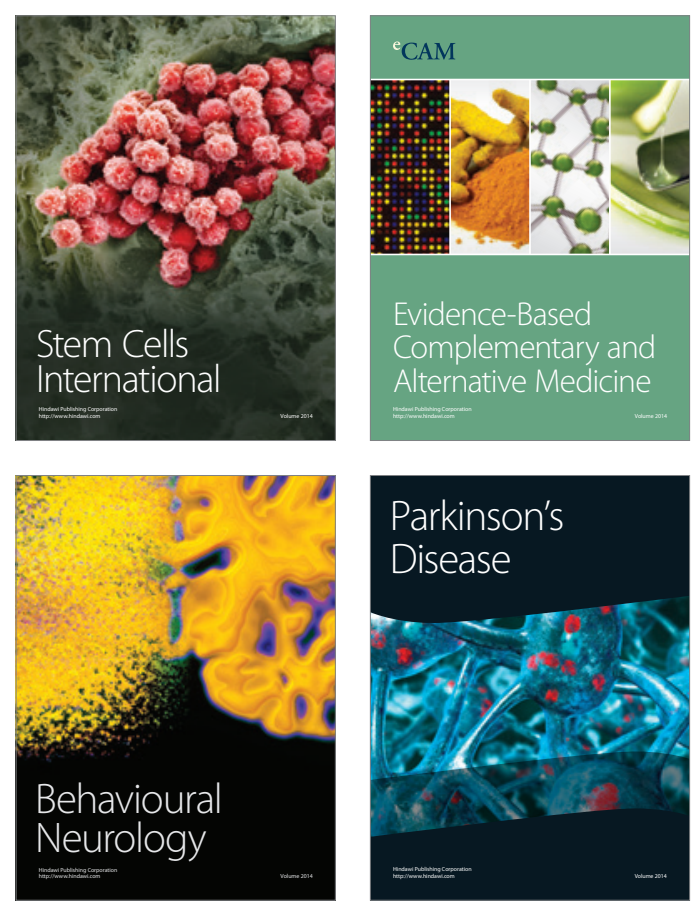

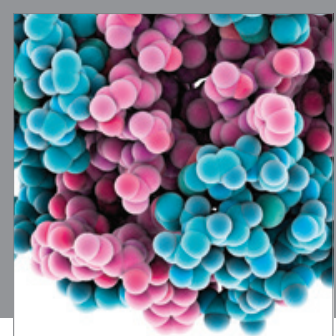

Journal of
Diabetes Research

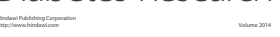

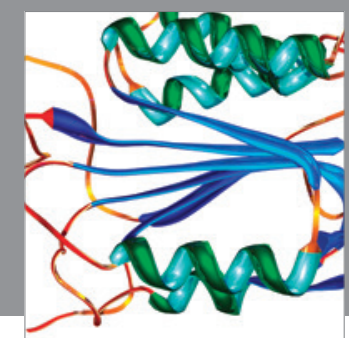

Disease Markers
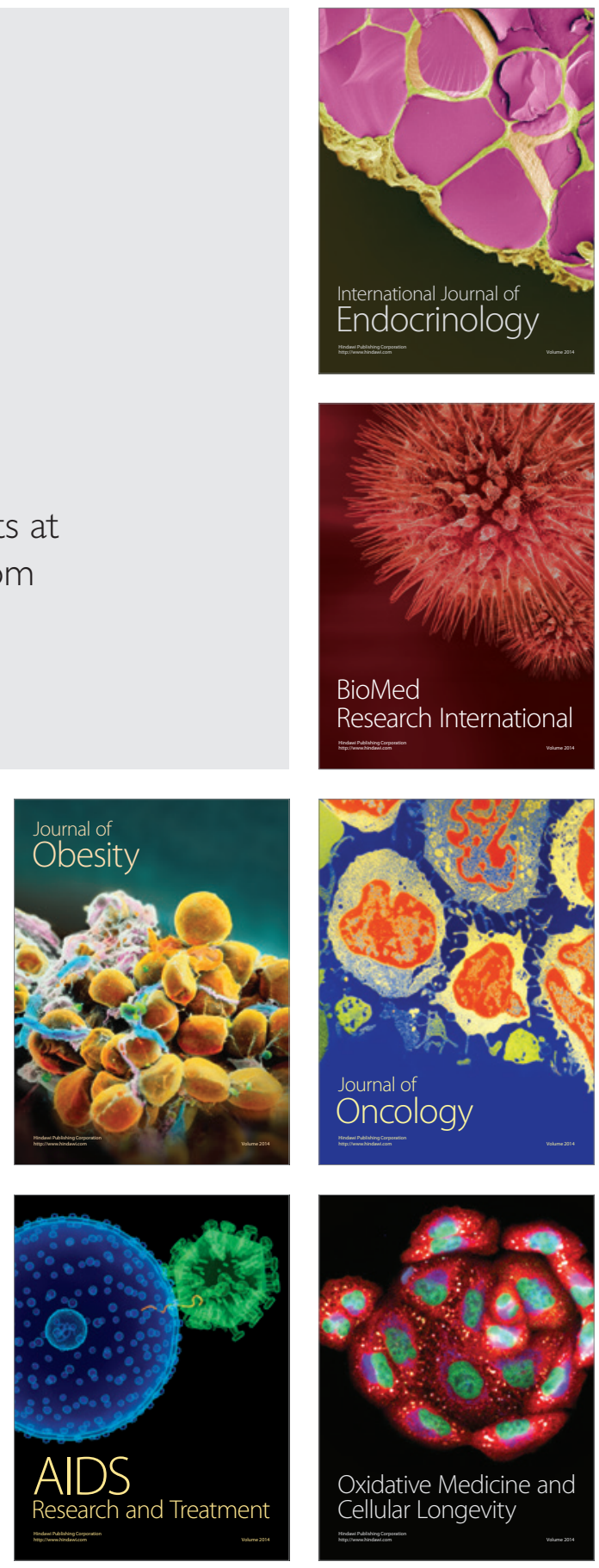